\title{
A CASE REPORT OF OSTEOCHONDROMA OF MANDIBLE
}

\author{
Bhaven M. Shah ${ }^{1}$
}

\section{HOW TO CITE THIS ARTICLE:}

Bhaven M Shah. "A case report of osteochondroma of mandible". Journal of Evolution of Medical and Dental Sciences 2013; Vol2, Issue 40, October 07; Page: 7739-7742.

ABSTRACT: Osteochondroma / osteocartilagenous exostosis is a cartilage-capped exophytic lesion that arises from the cortex of bone. It constitutes $20-50 \%$ of all benign tumours and $10-15 \%$ of all bone tumours, but it is actually a developmental lesion rather than a true neoplasm. An osteochondroma in the maxillo- facial region, however, is rare. In this anatomical area, the tumour is most often reported in association with the coronoid process. Osteochondroma of the mandibular condyle is rare and only 37 documented cases have so far been reported in the English language literature. I report a case of osteochondroma of the mandibular condyle and describe the characteristic OPG and CT features of this condition.

INTRODUCTION: Osteochondroma (OC) of the mandibular condyle is a relatively rare condition that causes a progressive enlargement of the condyle, usually resulting in facial asymmetry, temporomandibular joint (TMJ) dysfunction, and malocclusion. Radiographically, there is a unilaterally enlarged condyle usually with an exophytic outgrowth of the tumor from the condylar head. I present a case of a left mandibular condylar OC that created a malocclusion, and TMJ Dysfunction.

CASE PRESENTATION: A 42 year old male patient came with complains of Gradual onset of restricted mouth opening. On examination, there was restricted mouth opening. No significant facial asymmetry noted. No evidence of palpable lesion noted on left side of face. The patient underwent OPG which showed large Osteolytic lesion in the region of left mandibular condyle (Figure 1). The lesion was fairly well circumscribed with thin cortex. Left mandibular condyle was not seen separately. The patient was adviced CT scan for further evaluation. Saggital and coronal CT images revealed a large globular, mixed bone density condylar mass arising from left mandibular condyle (Figures 2 and 3). The lesion was extending superiorly into the articular fossa, but the cortical bone of the fossa was intact. The cortex of the tumor was continuous with that of the normal condylar bone. The pattern of the trabecular bone of the tumor blended with the intramedullary host bone. There was an evidence of pseudoarthosis formation with greater wing of sphenoid bone with thinning of greater wing. The lesion was reaching up to pterygoid process with compression and thinning of pterygoid process. Mandibular condyle was displaced laterally by the lesion. Tumor excision was performed. Histopathologic examination revealed a nodular lesion having cartilaginous cap and mature bone tissue beneath, consistent with osteochondroma.

DISCUSSION: Osteochondroma is a benign tumour of the appendicular skeleton arising from the metaphyseal regions of long bones, most commonly seen in the second and third decade of life. Osteochondromas are not common in the mandibular condylar region. Review of the literature indicates that patients with these tumours present mainly in the fourth decade with mean age of 39.7 years and a male to female ratio of 1:1.5. Clinical findings associated with osteochondroma of 
the condyle are primarily a palpable, painless temporomandibular area mass with lower facial asymmetry and prognathic deviation. In some cases, the mass in the condylar region may cause severe pain, hypomobility, and clicking and locking of the temporoman- dibular joint, as well as headaches and cervical pain. The pathogenesis of osteochondromas of the mandibular condyle is speculative. Trauma and inflammation have been implicated as either initiating or predisposing factors. Lesions consistently arise from the anteromedial surface of the condylar process at the site of attachment of the lateral pterygoid muscle. The tumours are thought to develop from the tendinous attachment of this muscle, similar to the tendency of long bone osteochondromas to arise at the tendinous attachments. In the mandibular condylar region, the radiographic presentation of the osteochondroma is that of an enlarged sclerotic mass that can reach gigantic proportions. Osteochondromas are found most often on the medial aspect of the mandibular condyle (52\%), followed by an anterior location (20\%), and rarely in the lateral or superior positions (, 1\%). Pre-operative CT plays an invaluable role in the treatment planning of these tumours. The tumour is seen as a bony outgrowth arising from the condylar neck. CT clearly depicts the continuation of the cortex and medulla of the parent bone with that of the tumour, a feature considered diagnostic of osteochondromas. ${ }^{1}$ Although CT is not the best imaging modality for evaluation of the uncalcified cartilage cap, calcified cartilage can be well depicted on CT. CT also delineates the soft-tissue alterations secondary to the growth, which in our patient included fatty infiltration and muscular atrophy of the masticator muscles. Buoncristiani et al reported fatty degeneration of the ipsilateral lateral pterygoid muscle on MRI in a case of osteochondroma arising from the glenoid fossa. This was speculated to be a functional response of the muscle to aberrant mandibular position. ${ }^{2}$ There are no reports that discuss similar soft-tissue changes in the masticatory muscles on CT, and the identification of this sign on CT is a new and useful finding. Histologically, the osteochondroma has a layer of thickened, cellular periosteum, deep to which lies a sheet of proliferating chondrocytes. Under this cartilaginous cap is a zone of ossification resulting in the formation of cancellous bone. Osteochondroma of the mandibular condyle must be distinguished from unilateral condylar hyperplasia. The latter is manifested clinically and radiographically as an enlarged condylar process $^{3}$, whereas the osteochondroma usually shows a globular projection extending from the margins of the condylar head with the normal outline of the condylar head being maintained. CT plays a decisive role in differentiating the two entities in that the osteochondroma is seen as a growth arising from the morphologically normal condyle, while condylar hyperplasia is seen as enlargement of the condylar process.

CONCLUSION: Mandibular osteochondroma, though a rare entity, should be considered in the differential diagnosis of masses in the region of the temporomandibular joint. Panoramic radiographs at best can be considered as a screening modality in the detection of these lesions. A CT examination should be performed in all cases of suspected osteochondroma of the mandibular condyle.

\section{REFERENCE:}

1. Murphey MD, Choi JJ, Kransdorf KJ, Fleming DJ, Gannon HF. Imaging of osteochondroma: varients and complications with radilogic-pathologic correlation. Radiographics 200: 20: 1407-1434 
2. Buoncristiani RD, Casagrande A, Felsenfeld AL. Osteochondroma of the glenoid fossa: occurrence in an atypical location. J Oral Maxillofac Surg 2003; 61: 134-137.

3. Saito T, Utsunomiya T, Furutani M, Yamamoto H. Osteochondrome of the mandibular condyle: a case report and review of literature. J Oral Sci 2001; 43: 293-297.

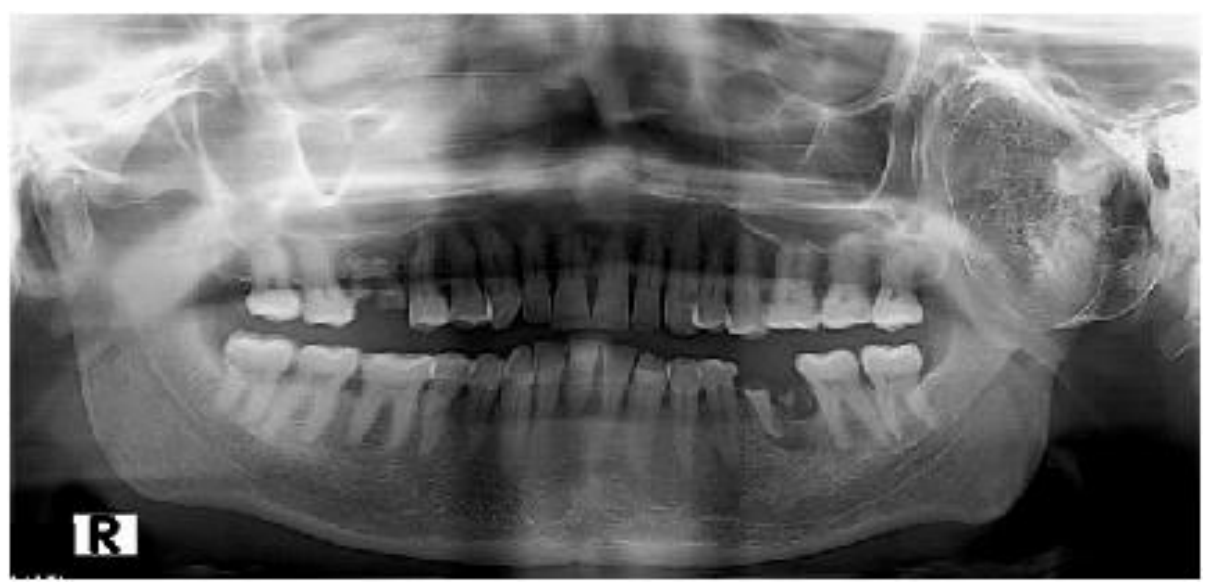

Figure 1: Orthopentogram showing expansile osteolytic lesion noted in mandibular condyle on left side.

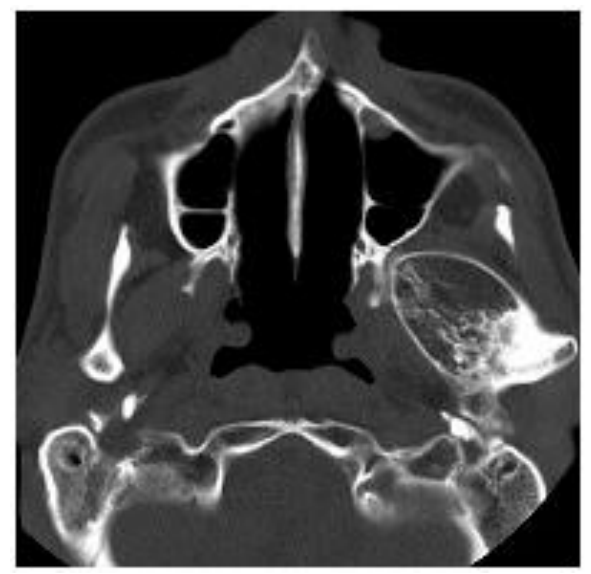

Figure 2 : Axial non contrast CT scan showing expansile lesion in left side mandibular condyle with continuation of medulla of mandible with the lesion. 


\section{CASE REPORT}

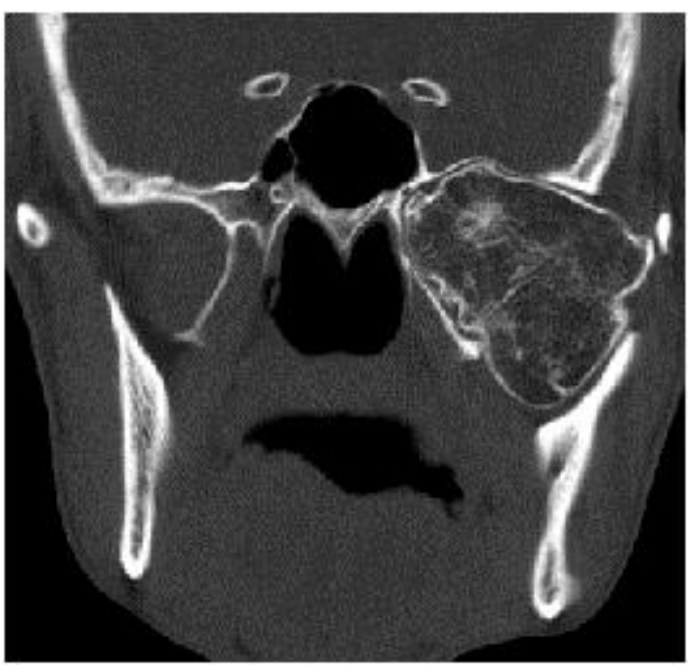

Figure 3: Coronal CT scan reveals pseudoarthrosis formation between the expansile lesion and sphenoid bone with remodeling of the sphenoid bone. No cartilage cap noted.

\section{AUTHORS:}

1. Bhaven M. Shah

\section{PARTICULARS OF CONTRIBUTORS:}

1. Assistant Professor, Department of RadioDiagnosis, Gujarat Adani Institute of Medical Science.

\section{NAME ADDRESS EMAIL ID OF THE} CORRESPONDING AUTHOR:

Dr. Bhaven M. Shah, Plot No. 32, Ashapura Park, Mundra Road, Nr Leva Patel Doctor Quarters, BHUJ, Kutch - 370001.

Email-bhavenshaw83@gmail.com

Date of Submission: 08/08/2013.

Date of Peer Review: 12/08/2013.

Date of Acceptance: 26/08/2013.

Date of Publishing: 04/10/2013 